\title{
Apports de nutriments et développement phytoplanctonique en baie de Seine
}

\author{
Alain AMINOT, Jean-François GUILLAUD, Françoise ANDRIEUX-LOYER, \\ Roger KÉROUEL, Philippe CANN
}

Ifremer-centre de Brest, département écologie côtière, BP 70, 29280 Plouzané, France

(Revisé le 19 mars 1998, accepté le 27 mars 1998)

Abstract - Nutrients and phytoplanktonic growth in the Bay of Seine, France. As part of the Programme National d'Océanographie Cottière, the nutrient dynamics of the Bay of Seine were studied between 1992 and 1994 in order to complement work on ecological modelling. Firstly, the River Seine's nutrient fluxes were established: $80000-130000 \mathrm{ta}^{-1}$ of dissolved inorganic nitrogen, $6400-8400 \mathrm{ta}^{-1}$ of dissolved phosphorus and $20000-77000 \mathrm{ta}^{-1}$ of dissolved silicium. Estuarine processes were taken into account. Consequences of nutrient loading for the bay were then evaluated at the pelagic level (nutrient and chlorophyll enrichments) and the benthic level (distribution of various phosphorus fractions in superficial sediments). The large continental inputs always induce concentration gradients in the water from the mouth of the river to the northwest of the bay. The northward spreading of fine particulate matter controls the distribution of adsorbed phosphate and iron-aluminium bound phosphate in sediments. In contrast, calcium bound phosphate, the main fraction in sediments, is not affected by river imputs. Organic phosphorus in sediments is related to phytoplankton blooms, with increasing concentrations during productive periods; afterwards the fast recycling prevents sedimentary accumulation.

The nutrient depletions observed beyond the turbid plume during spring 1992 enabled the calculation of nutrient uptake rates, and the comparison of these rates with phytoplankton biomass (chlorophyll $a+$ phaeopigments). Ratios of nutrient consumption to pigment concentration were estimated at $1 \mu \mathrm{mol} \mu \mathrm{g}^{-1}$ for nitrogen, 0.05 for phosphorus and 0.5 for silicate. These values, as N/P and $\mathrm{Si} / \mathrm{N}$ uptake ratios (respectively 17.5 and 0.4 ), were similar to usual values. Elsevier, Paris

\section{nutrient / chlorophyll / uptake ratio / Bay of Seine}

Résumé - Cette étude des nutriments en baie de Seine a été réalisée dans le cadre du Programme national d'océanographie côtière, entre 1992 et 1994, en parallèle avec les travaux de modélisation écologique. Les flux de nutriments de la Seine ont tout d'abord été évalués: $80000-130000 \mathrm{t} \mathrm{a}^{-1}$ d'azote minéral dissous, $6400-8400 \mathrm{ta}^{-1}$ de phosphore dissous et 20000-77000 $\mathrm{ta}^{-1}$ de silicium dissous. Le rôle de l'estuaire sur ces flux ainsi que les conséquences de ces apports sur la baie ont ensuite été mesurés au niveau pélagique (enrichissement en nutriments et en chlorophylle) et au niveau benthique (répartition des formes de phosphore dans les sédiments superficiels). Ainsi, les forts apports continentaux se traduisent toujours dans l'eau par des gradients globalement orientés au nord-ouest depuis l'embouchure de la Seine. Pour le sédiment superficiel, la dispersion du matériel fin plutôt en direction nord à nord-est commande la répartition du phosphate adsorbé et du phosphate lié au fer et à l'aluminium. Le phosphate apatitique, fortement majoritaire, n'est pas significativement affecté par les apports. Le phosphore organique est sensible aux développements phytoplanctoniques : son niveau augmente durant la période productive mais il ne s'accumule pas, apparaissant donc rapidement recyclé.

Après la mise en évidence, au printemps 1992, de déficits de nutriments au-delà du panache turbide de la Seine, des consommations ont été calculées et comparées aux concentrations d'un traceur de biomasse (chlorophylle $a+$ phéopig-

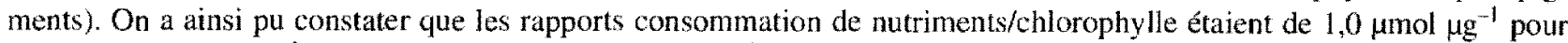
l'azote, $0,05 \mu \mathrm{mol} \mu \mathrm{g}^{-1}$ pour le phosphore et $0,5 \mu \mathrm{mol} \mu \mathrm{g}^{-1}$ pour le silicate. Ces valeurs, ainsi que les rapports de consommation calculés $(17,5$ pour N/P, et 0,4 pour $\mathrm{Si} / \mathrm{N})$ sont très proches des valeurs classiquement admises. $\odot$ Elsevier, Paris

nutriment / chlorophylle / rapport de consommation / baie de Seine 


\section{INTRODUCTION}

La baie de Seine, d'une superficie de près de $4000 \mathrm{~km}^{2}$, est largement ouverte au nord, sur la Manche; la profondeur n'y dépasse guère une trentaine de mètres et le marnage maximum est de l'ordre de $7 \mathrm{~m}$. Située au débouché d'un bassin versant de $75000 \mathrm{~km}^{2}$ soumis à une pression agricole et urbaine représentant respectivement $20 \%$ et $30 \%$ de l'activité nationale [15], la baie de Seine est enrichie en éléments nutritifs, particulièrement en azote et phosphore.

Des poussées phytoplanctoniques intenses y ont été observées en périodes printanière et estivale depuis de nombreuses années [28, 32]. Entre 1975 et 1988, Belin et al. [10] ont recensé 46 cas d'eaux colorées, dus essentiellement aux développements de diatomées puis de dinoflagellés. Cependant les travaux sur les apports et le comportement des eléments nutritifs en baie de Seine restent limités $[1,3,16]$.

L'objet du présent travail est de synthétiser les données de nutriments acquises dans le cadre du Programme national d'océanographie côtière (PNOC). Tout d'abord il s'agit de déterminer l'état nutritionnel de la baie tel qu'il ressort des flux continentaux et de leurs répercussions sur les concentrations dans la baie. En outre, il est nécessaire de réunir des éléments permettant une meilleure approche par modélisation de l'eutrophisation côtière. Ce second aspect recouvre l'étude du développement du phytoplancton et des rapports d'assimilation des nutriments. Toutefois, ce travail se voulant essentiellement synthétique. l'interprétation n'a pas été étendue aux formes spécifiques de l'azote minéral dissous. Des données complémentaires sur l'ammonium sont disponibles par ailleurs [2].

\section{MATÉRIEL ET MÉTHODES}

Huit campagnes ont été réalisées : 6-8 novembre 1991 (estuaire seul), 17-19 février 1992, 24-30 avril 1992, 2-4 juin 1992, 29 juin-3 juillet $1992,25-28$ avril 1994, $22-$ 25 juin 1994 et 22-25 septembre 1994.

L'eau a été prélevée à l'aide de bouteilles en PVC de $8 \mathrm{~L}$, de type Niskin. Les échantillons sont préfiltrés directement en sortie de la bouteille de prélèvement, à $200 \mu \mathrm{m}$ pour le matériel particulaire et à $10 \mu \mathrm{m}$ pour les nutriments. Le matériel particulaire est aussitôt filtré à bord. Les filtres chargés ainsi que les échantillons pour les nutriments (excepté silicate) sont immédiatement placés au congélateur pour une analyse ultérieure au laboratoire. Les échantillons pour la mesure du silicate sont filtrés à 1,2 $\mu \mathrm{m}$ (membranes en acétate de cellulose), empoisonnés au chlonure mercurique ( $20 \mathrm{mg} \mathrm{L}^{-1}$ ), puis maintenus à l'obscurité, à température ambiante, jusqu'à l'analyse. Les nutriments ont été dosés à l'aide d'un Autoanalyzer II Technicon [34]. La salinité est mesurée sur l'échelle pratique de salinité (PSS78) avec un salinomètre Guildline Portasal. La chlorophylle et les phéopigments sont mesurés par spectrophotométrie [24].

Les sédiments ont été prélevés à la benne Shipek. Seule la couche superficielle $(-2 \mathrm{~cm})$ a été recueillie. Les formes de phosphore sédimentaire ont été obtenues par extractions successives, selon le schéma classique de Williams et al. [38], modifié par Psenner et al. [30]. Ce protocole a été décrit antérieurement $[1,4]$.

Les concentrations dans l'eau douce et le calcul des flux sont issus des données de la cellule antipollution de la Seine (1977-1994). Les mesures bimensuelles sont effectuées à Caudebec, situé à $50 \mathrm{~km}$ de l'embouchure (Le Havre). Les flux journaliers résultent du produit des débits par les concentrations : les flux annuels ( $\mathrm{Fa}$ ) en tonnes par an ont été évalués par la relation de Walling et Webb [37]: $\mathrm{Fa}=\mathrm{k} \cdot \mathrm{Q}_{\mathrm{m}} \cdot\left(\Sigma \mathrm{Q}_{\mathrm{i}} \mathrm{C}_{\mathrm{i}} / \Sigma \mathrm{Q}_{\mathrm{i}}\right)$, avec $\mathrm{Q}_{\mathrm{m}}=$ débit moyen annuel $\left(\mathrm{m}^{3} \mathrm{~s}^{-1}\right), Q_{\mathrm{j}}=$ débit instantané $\left(\mathrm{m}^{3} \mathrm{~s}^{-1}\right)$, $C_{i}=$ concentration instantanée $\left(m g L^{-1}\right), k=$ coefficient correcteur d'unité.

\section{RESULTATS ET DISCUSSION}

\subsection{Concentrations et flux de la Seine}

Les évolutions des débits et des concentrations en nutriments durant les trois années d'étude (1992 à 1994) sont illustrées sur la figure 1 . Les variations saisonnières des débits sont fortes, avec des niveaux exceptionnellement faibles en janvier et février 1992 et de fortes crues au cours de l'hiver 1993-1994. En 1992 le débit moyen est inférieur de $12 \%$ au débit moyen interannuel $\left(400 \mathrm{~m}^{3}\right.$ $\mathrm{s}^{-1}$ ) tandis qu'en 1994 il lui est supérieur de $60 \%$.

\subsubsection{Concentrations}

Les concentrations en azote minéral dissous sont élevées et relativement stables au cours de l'année (400$\left.700 \mu \mathrm{mol} \mathrm{L}^{-1}\right)$. L'azote organique total, réparti grossièrement par moitié sous formes particulaire et dissoute, représente en moyenne $12 \%$ de l'azote total ; ses teneurs 

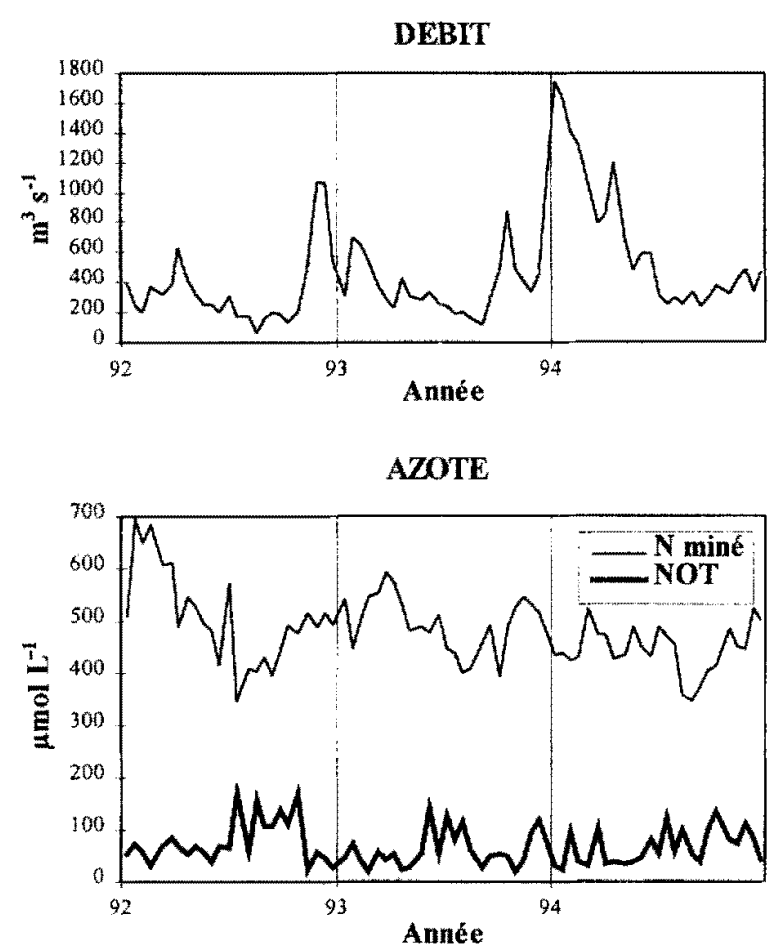

PHOSPHORE

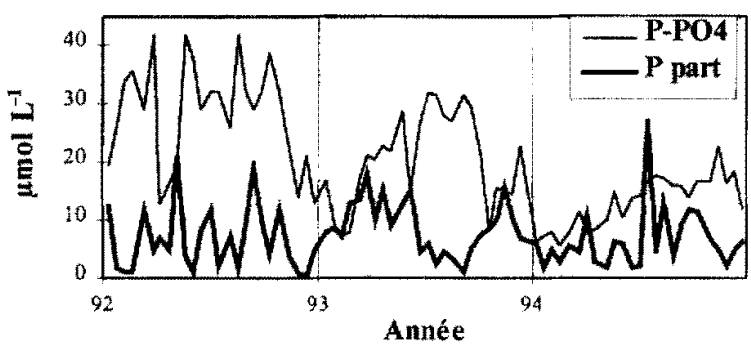

SILICATE

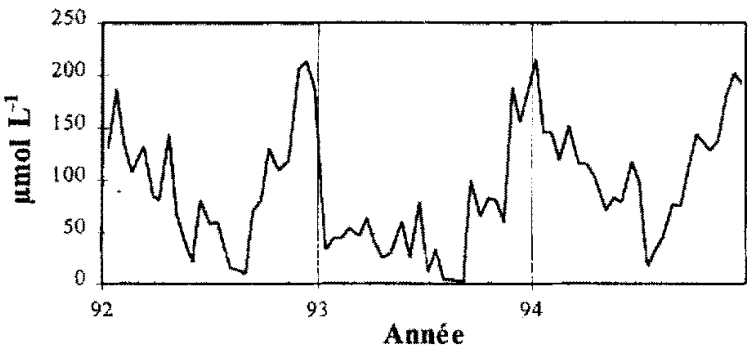

fluctuent et semblent augmenter durant les étiages prononcés de 1992 et 1993. Les concentrations en phosphate, êlevées et très variables $\left(10-50 \mu \mathrm{mol} \mathrm{L}^{-1}\right)$, varient en fonction inverse du débit car les sources ponctuelles (délivrant un flux constant de phosphate) dominent par rapport au phénomène de lessivage sur le bassin versant [1]. Les données de la Cellule antipollution de la Seine (1977-1994) montrent que les teneurs en phosphore particulaire dépendent significativement des concentrations en matières en suspension et représentent en moyenne $28 \%$ du phosphore total. Le silicate varie très fortement entre des valeurs hivernales atteignant $200 \mu \mathrm{mol} \mathrm{L}^{-1}$ et un quasi épuisement estival, dû à la consommation phytoplanctonique; il n'existe pas de relation simple entre le silicate et le débit [25].

\subsubsection{Flux}

Les flux annuels d'azote minéral dissous et de silicate ont été nettement plus élevés durant l'année 1994, beaucoup plus humide que les deux années précédentes (tableau $I$ ),

Tableau I. Débit moyen de la Seine et flux d'éléments nutritifs dissous à Caudebec. Note : le flux de phosphore est déterminé sur la base du phosphate.

Table I. Mean flow of the River Seine and dissolved nutrient fluxes at Caudebec. Note: the phosphorus flux is based on phosphate.

\begin{tabular}{lcccc}
\hline Année & $\begin{array}{c}\text { Débit } \\
\left(\mathbf{m}^{3} \mathbf{s}^{-1}\right)\end{array}$ & \multicolumn{3}{c}{ Flux $\left(\mathrm{t} \mathrm{a}^{-\mathbf{1}}\right)$} \\
\cline { 3 - 5 } & & Azote minéral & Phosphore & Silicium \\
\hline 1992 & 348 & 81000 & 8400 & 40000 \\
1993 & 408 & 82000 & 6400 & 20000 \\
1994 & 669 & 132000 & 6800 & 77000 \\
\hline
\end{tabular}

montrant l'importance du lessivage des sols pour ces deux nutriments. Les flux de phosphate, au contraire, sont restés relativement stables. Les flux d'azote organique total sont faibles par rapport au flux d'azote total (de l'ordre de $10 \%$ ) alors que les flux de phosphore particulaire représentent près de $30 \%$ des apports en phosphore total. On peut rappeler l'évolution à long terme des flux d'azote minéral dissous qui, sur la période 1977-1994,

Figure 1. Débit de la Seine et concentrations en azote (N miné : azote total minéral dissous; NOT : azote organique total), en phosphore (P-PO ${ }_{4}$ : phosphate; $P$ part : phosphore particulaire) et en silicate à Caudebec de 1992 à 1994.

Figure 1. Flow of the River Seine and concentrations of nitrogen ( $N$ miné: dissolved inorganic nitrogen; NOT: total organic nitrogen), phosphorus (P-PO 4 : phosphate: P part: particulate phosphorus) and silicate at Caudebec from 1992 to 1994. 
ont augmenté en moyenne de 2,2\% par an [25]. Les flux de phosphate ont, sur la même période, diminué de $4 \%$, du fait de la réduction des rejets industriels rouennais. Les flux de silicate sont restés globalement stables.

\subsection{Comportement des nutriments dans l'estuaire}

Au cours des campagnes de 199 I à 1994, plusieurs incursions on été effectuées dans l'estuaire de la Seine jusqu'à des salinités très faibles. Les mesures de nutriments faites à ces occasions illustrent leur comportement au cours du transit estuarien (figure 2). Pour l'azote minéral total dissous, les droites tracées pour certaines campagnes montrent que le transit est conservatif jusqu'à une salinité d'environ 30, correspondant grossièrement à l'embouchure de l'estuaire. Les fluctuations des concentrations en azote à la source continentale $\left(400-700 \mu \mathrm{mol} \mathrm{L}{ }^{1}\right)$ corroborent les données de la cellule antipollution de la Seine (1977-1994). Néanmoins, ces fluctuations sont assez lentes en regard du temps de séjour des eaux dans l'estuaire (moins de $10 \mathrm{j}$ pour un débit supérieur a $200 \mathrm{~m}^{3} \mathrm{~s}^{-1}$ [6]), c'est pourquoi la dilution se traduit par une relation linéaire avec la salinité. En ce qui concerne phosphate et silicate, les variations extrêmement fortes et rapides des concentrations en Seine (figure $l$ ) font que l'hypothèse d'état stationnaire n'est plus vérifiée, ce qui invalide la référence à une droite de dilution pour évaluer la conservativité de ces nutriments dans l'estuaire [23]. Pour le phosphate, la dilution reste toutefois presque linéaire, excepté en avril et juillet 1992 où les concentrations s'abaissent et s'élèvent, respectivement, aux basses salinités. En juillet, compte tenu de la relation phosphatedébit, l'accroissement de la concentration en eau dessalée paraît dû à la baisse rapide du débit fluvial $(280$ à $110 \mathrm{~m}^{3} \mathrm{~s}^{-1}$ ), dans les jours précédant l'observation. En avril, à débit quasi constant, nous ne disposons pas de données complémentaires permettant une interprétation satisfaisante. D'une manière générale, les concentrations en phosphore de la Seine sont très élevées et leurs variations peuvent masquer des phénomènes de moindre intensité, tels que les échanges avec le matériel particulaire. En silicate, les concentrations en eau douce variant considérablement, il est difficile de mettre en évidence des processus estuariens. La faible courbure (figure 2) des relations de dilution tendrait toutefois à confirmer l'hypothèse d'une dissolution de matériel particulaire (algues siliceuses) dans l'estuaire [16].

Peu d'informations sont disponibles sur le devenir dans l'estuaire des éléments nutritifs transportés sous forme
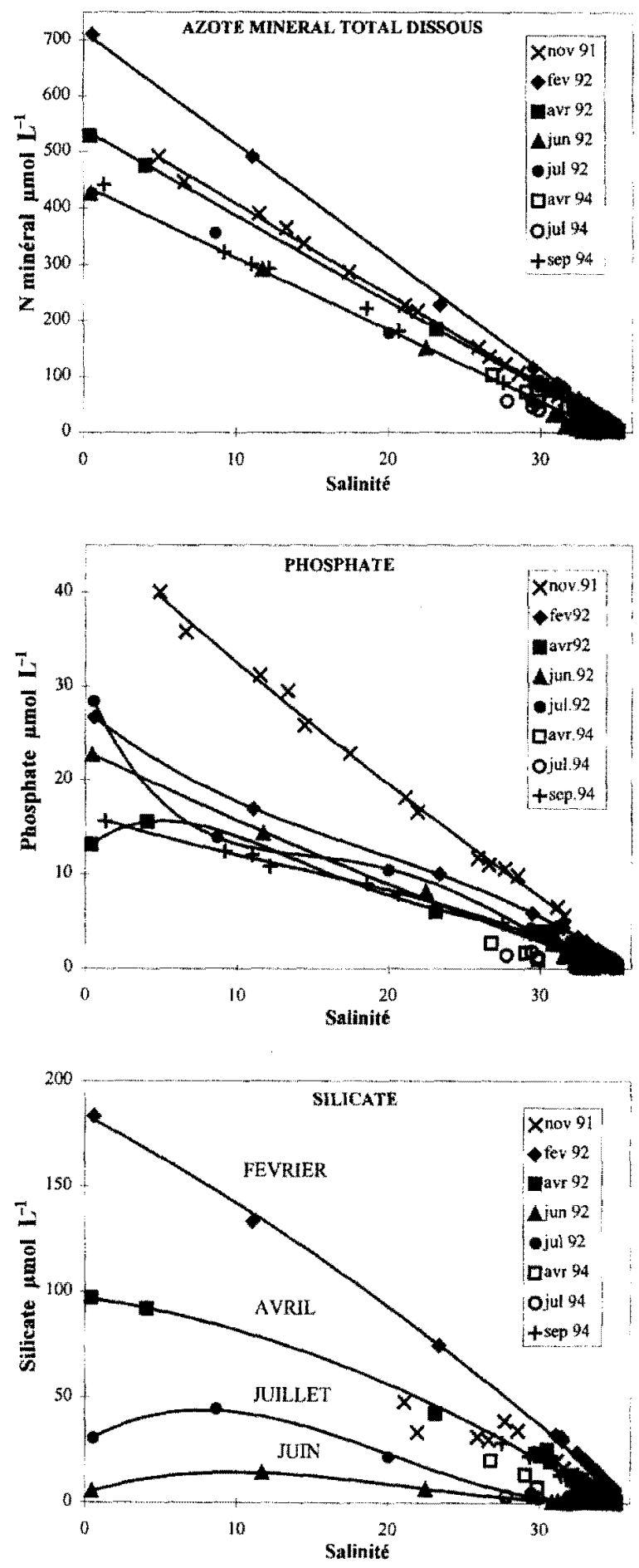

Figure 2. Relations nutriment-salinité dans l'estuaire de la Seine entre novembre 1991 et septembre 1994.

Figure 2. Nutrient-salinity relationships in the Seine estuary from November 1991 to September 1994. 
particulaire. Leurs concentrations dans la masse d'eau sont très variables et fonction de la dynamique des particules, celles-ci étant déposées et remises en suspen. sion au cours du cycle de marée, et parfois évacuées massivement dans la baie lors des crues [8]. C'est le taux d'élément nutritif dans le matériel particulaire qui doit être examiné. Pour l'azote, les données de Boust [12] montrent que son pourcentage décrôt de moitié environ de l'amont vers l'aval pour se situer à $0,2-0,3 \% \quad\left(150-200 \mu \mathrm{mol} \mathrm{g}^{-1}\right)$ en sortie d'estuaire. Comme le rapport $\mathrm{C} N$ decroît simultanément, la dégradation du matériel organique peut expliquer cette baisse; ceci indiquerait que l'azote particulaire évacué de l'estuaire est plus réfractaire et ne contribue pas significativement à l'enrichissement en nutriments. Par ailleurs, la partie minéralisée ne représentant que quelques pourcents de l'apport azoté de la Seine, son incidence sur l'azote dissous ne peut pas être mise en évidence. Pour le phosphore, une étude précédente [1] a montré que sa concentration dans le matériel particulaire restait stable sur tout l'estuaire $\left(70-90 \mu \mathrm{mol} \mathrm{g}^{-1}\right)$, avec environ $50 \%$ de phosphore potentiellement biodisponible.

Aucune donnée n'est disponible sur le silicium particulaire, dont la fraction biogénique est intéressante. Dans l'hypothèse maximaliste où toute la biomasse phytoplanctonique fluviale serait constituée de diatomées, avec un rapport Si/pigments d"environ $0,5 \mu \mathrm{mol} \mu \mathrm{g}^{-1}$ (voir ci-dessous) on calcule, en moyenne pour l'eau douce, des concentrations correspondantes de silicium particulaire d'environ $10 \mu \mathrm{mol} \mathrm{L}^{-1}$ en hiver, de 20 à $40 \mu \mathrm{mol} \mathrm{L}^{-1}$ au printemps et en automne, et jusqu'à $50 \mu \mathrm{mol} \mathrm{L} \mathrm{L}^{-1}$ en juillet, d'après les données de pigments de la cellule antipollution de la Seine (1977-1994). Ce résultat est en bon accord avec la courbure positive des relations silicate-salinité (figure 2) dont l'extrapolation linéaire à salinité nulle fait ressortir des suppléments d'environ $20 \mu \mathrm{mol} \mathrm{L} \mathrm{L}^{-1}$ en juin et $40 \mu \mathrm{mol} \mathrm{L} \mathrm{L}^{-1}$ en juillet, par rapport aux mesures de silicate dissous en eau douce.

Globalement, on peut considérer que l'estuaire n'affecte pas dans des proportions notables les flux d'éléments nutritifs de la Seine exportés dans la baie. Les évaluations effectuées à Caudebec, juste en amont de l'intrusion saline, représentent donc les apports en baie de Seine, sous réserve d'ajouter au silicium dissous la fraction particulaire biogénique.

\subsection{Influence des apports sur la baie}

L'influence des apports d'eau douce de la Seine sur la baie, qui se traduit par un gradient de salinité orienté du sud-est vers le nord-ouest, est d'autant plus étendue que les débits fluviaux sont élevés. Ainsi, l'isohaline 34, qui pénètre largement dans la moitié est de la baie en année sèche (figure 3), peut être repoussée à la limite nord-ouest lors des années humides [3]. La distribution des nutriments dans la baie est similaire à la répartition des salinités, notamment en hiver où, du fait de l'activité biologique réduite, les relations salinité-nutriments sont lineaires.

Les matières en suspension apportées par la Seine $(0,5$ à 1 million de tonnes par an [7]) se dispersent dans la masse d'eau, à la sortie de l'estuaire, selon un gradient proche de celui de la salinité (figure 3). Néanmoins, au niveau benthique, le matériel fin d'origine estuarienne se répartit majoritairement vers le nord, le long de la côte est de la baie [9].

Le phosphore particulaire véhiculé par les matières en suspension influence les concentrations dans les sédiments (figure 3). Les concentrations de phosphore sédimentaire total sont de 10 a $15 \mu \mathrm{mol} \mathrm{g}{ }^{-1}$ quelle que soit l'époque de l'année, une gamme relativement restreinte en dépit des apports de la Seine. Les répartitions du phosphate échangeable (P-éch) et du phosphate lié au fer et à l'aluminium ( $\mathrm{P}-\mathrm{Fe} / \mathrm{Al})$ sont similaires avec un gradient décroissant est-ouest bien marqué ; ces répartitions ont été retrouvées sans changement notable au cours des autres campagnes. L'orientation vers le nord des lignes d'égales concentrations reflète la décantation des particules fines expulsées de l'estuaire. Pour les phosphates de calcium (généralement apatitiques; P-Ca) les concentrations sont plus élevées (6 à $8 \mu \mathrm{mol} \mathrm{g}^{-1}$ ), stables dans le temps, et leur répartition est plus uniforme du fait d'une proportion significative de sédiments calcaires dans la baie. Les concentrations en phosphore organique ( $\mathrm{P}$-orga) sont basses et uniformes en février $\left(0,5-1 \mu \mathrm{mol} \mathrm{g}^{-1}\right)$ mais s"élèvent au cours de la période productive. Toutefois l'influence des apports de P-orga à l'embouchure n'est jamais visible du fait, soit d'une décantation plus lente, soit d'une dégradation rapide de la matière organique phosphorée [4]. Globalement on peut rappeler que les phosphates liés au calcium, au fer et à l'aluminium (soit environ les trois quarts du phosphore sédimentaire) ne sont pas biodisponibles dans les conditions physicochimiques de la baie de Seine [1]. 
Salinité
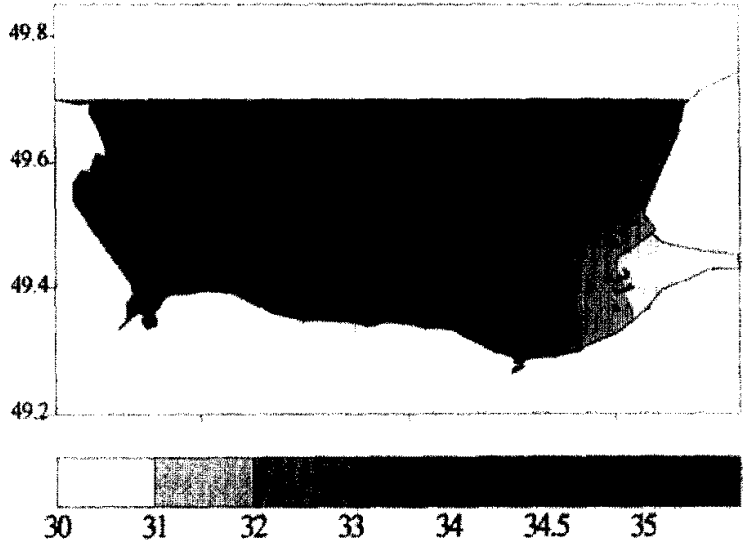

P-éch

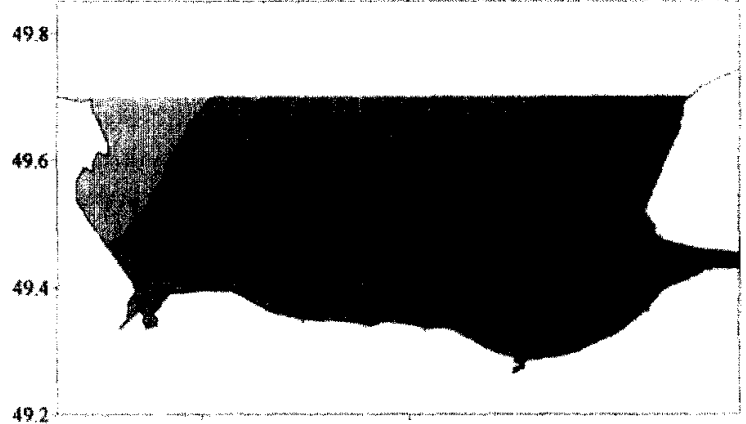

$$
\text { P-orga }
$$

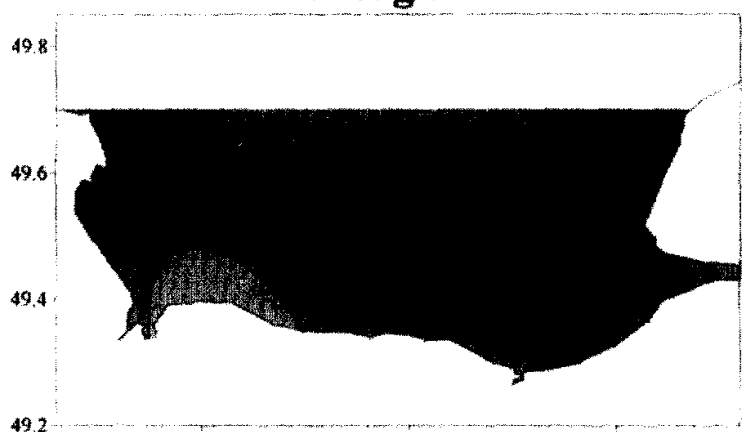

Turbidité

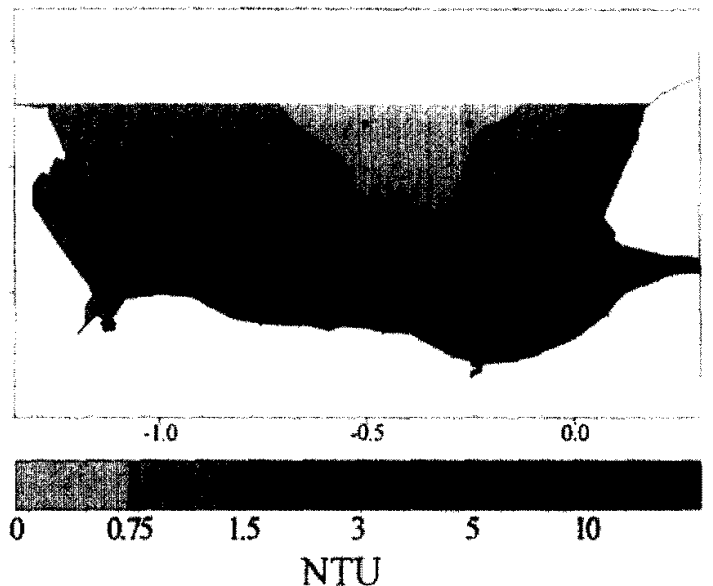

$\mathbf{P}-\mathbf{F e} / \mathbf{A l}$

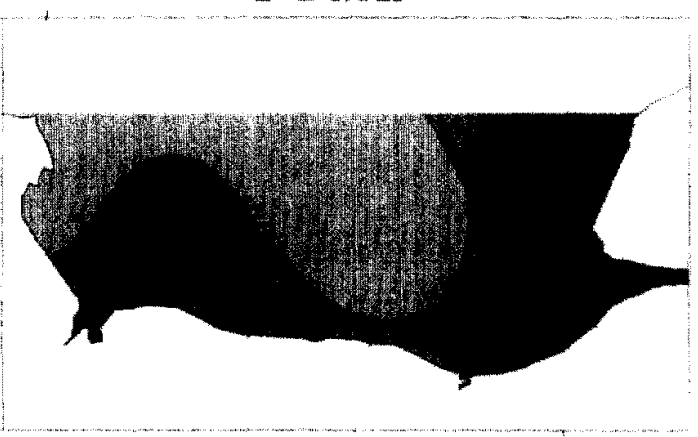

P-Ca

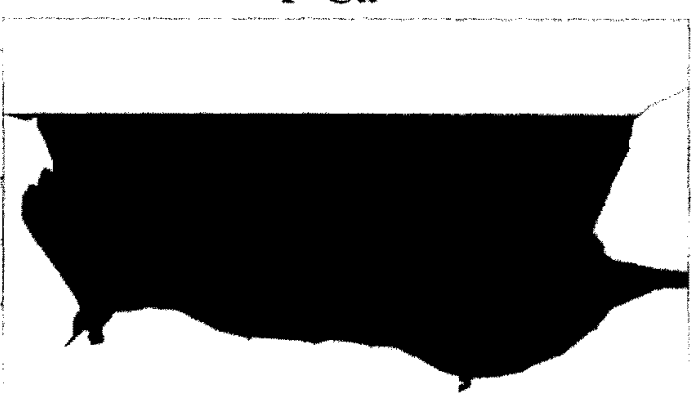

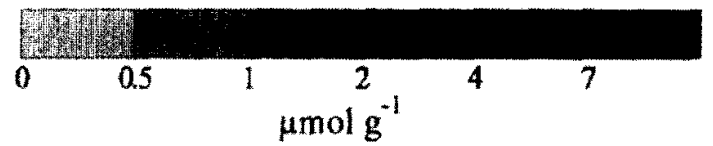

Figure 3. Distribution géographique de la salinité et de la turbidité dans l'eau de surface de la baie de Seine, ainsi que des formes de phosphore dans le sédiment superficiel, en février 1992.

Figure 3. Distribution pattern of salinity and turbidity in surface water of the Bay of Seine, and phosphorus forms in the upper sediment layer. in February 1992. 


\subsection{Biomasse phytoplanctonique et consommation des nutriments}

\subsubsection{Développement phytoplanctonique}

L'évolution de la chlorophylle $a$ dans la baie de février à juillet 1992 est illustrée sur la figure 4. Les concentrations en chlorophylle $a$ de février, très basses et quasi uniformes sur la baie $\left(0,5\right.$ à $\left.1 \mu \mathrm{g} \mathrm{L}^{-1}\right)$, sont associés à des pourcentages de phéopigments compris entre 40 et $70 \%$. En avril une forte poussée (jusqu'à $15 \mu \mathrm{g} \mathrm{L}^{-1}$ ) est observée au-delà du panache de la Seine, puis en juinjuillet la zone de forte concentration en chlorophylle $a$ est située contre la côte sud-est de la baie. Bien que nos observations n'aient pas pu en détecter, des niveaux de chlorophylle supérieurs à $40 \mu \mathrm{g} \mathrm{L}^{-1}$ sont mesurés plusieurs fois chaque année par le réseau national d'observation 1321 ; ils doivent être considérés comme très élevés, même en zone côtière.

Les poussées planctoniques se répercutent rapidement sur le phosphore organique sédimentaire, dont les concentrations s'accroissent significativement (jusqu "à $4 \mu \mathrm{mol} \mathrm{g}^{-1}$ ) dès le mois d'avril par rapport à la situation hivernale [4]. L'augmentation des teneurs, plus marquée dans la partie est de la baie, est à rattacher aux poussées phytoplanctoniques. Les zones des maximums de $\mathrm{P}$-orga suivent grossièrement le déplacement des maxima de chlorophylle $a$ et indiquent un faible déphasage entre poussée pélagique et impact benthique. Les teneurs élevées, observées en avril au nord-est de la baie, sont à relier aux poussées printanières initiales, mises en évidence indirectement par l'étude des consommations de nutriments (voir ciaprès). A aucun moment on ne voit d'accroissement significatif au droit de l'estuaire, situation attribuée à la dérive du matériel planctonique produit en cette zone. Le phosphore organique ne paraît pas s'accumuler durablement dans la baie, signe d'une régénération rapide avec retour au milieu pélagique.

\subsubsection{Evaluation des consommations de nutriments}

En avril 1992 et 1994, des concentrations de chlorophylle $a$ de 5 a $15 \mu \mathrm{g} \mathrm{L}^{-1}$ dans la plus grande partie de la baie montrent l'existence des premières poussées printanières. Simultanément de fortes chutes de nutriments sont observées dans les eaux de salinité supérieure à 33. Les résultats de l'année 1992, où des campagnes d'hiver et de printemps ont été effectuées, étaient particulièrement propices à l'évaluation de la consommation des nutriments par le phytoplancton. Pour le mois $\mathrm{d}^{*}$ avril on peut faire l'hypothèse que les premières poussées phytoplanctoniques dans la baie se développent sur le stock hivernal de nutriments issus des apports fluviatiles et marins, et que la production régénérée (c'est-à-dire se développant grâce à la reminéralisation du matériel planctonique antérieurement produit) est négligeable.

La consommation d'un nutriment en un point de la baie correspond à la différence entre, d'une part, la concentration théorique de ce nutriment résultant du simple mélange des eaux d'origines marine et continentale et, d'autre part, la concentration réellement observée. La droite de dilution utilisée pour le calcul des déficits est tracée entre deux points-sources (amont et aval). Comme référence amont nous avons choisi une station qui a une salinité de 32,1 et qui est peu perturbée par l'activité biologique (chlorophylle $a=0,9 \mu \mathrm{g} \mathrm{L}^{-1}$ ). Pour la référence aval en azote et en phosphore (salinite 35) nous avons choisi les concentrations hivernales (février 1992) non affectées par la consommation phytoplanctonique et donc parfaitement corrélées à la salinité. Ces concentrations sont corroborées par celles trouvées dans les eaux océaniques pénétrant en Manche en hiver $[5,14,22,27,33]$. Pour le silicate, la référence marine calculée à l'aide des mesures de février $1992\left(\mathrm{Si}=6 \mu \mathrm{mol} \mathrm{L}^{-1}\right)$ conduisait à une incohérence, à savoir des déficits élevés de ce nutriment associés à des déficits d'azote et de phosphore quasi nuls, en l'absence de chlorophylle a. La référence marine calculée, non conforme aux valeurs hivernales de silicate mesurées précédemment ( 3 à $4,5 \mu \mathrm{mol} \mathrm{L}^{-1}$ ) apparaît trop élevée. Nous avons retenu, pour la référence marine en silicate, la valeur hivernale moyenne des eaux pénétrant en Manche, soit 3,7 $\mu \mathrm{mol} \mathrm{L}$.

\subsubsection{Relations entre la biomasse phytoplanctonique et les deficits de nutriments}

Les déficits de nutriments ont été comparés à un paramètre représentatif de la biomasse phytoplanctonique totale, la somme chlorophylle $a+$ phéopigments (figure 5). Tous les prélèvements de la campagne d'avril (pleine et basse mer, surface et fond) dont la salinité est supérieure à 32 ont été utilisés pour cette analyse. Il apparaît que l'augmentation des déficits est bien reliée, notamment pour l'azote minéral total dissous et le phosphate, à l'accroissement du marqueur de biomasse phytoplanctonique.

En azote et en phosphate, un groupe de stations à faibles concentrations de chlorophylle se distingue par des déficits plus importants que ceux observés dans le reste de la 


\section{Chlorophylle $a$}

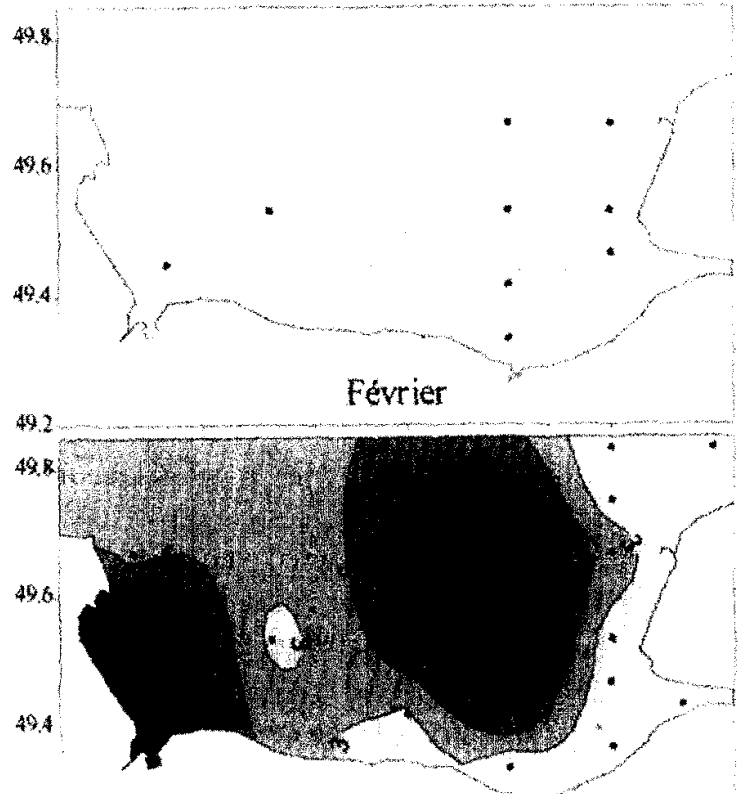

Fin arril
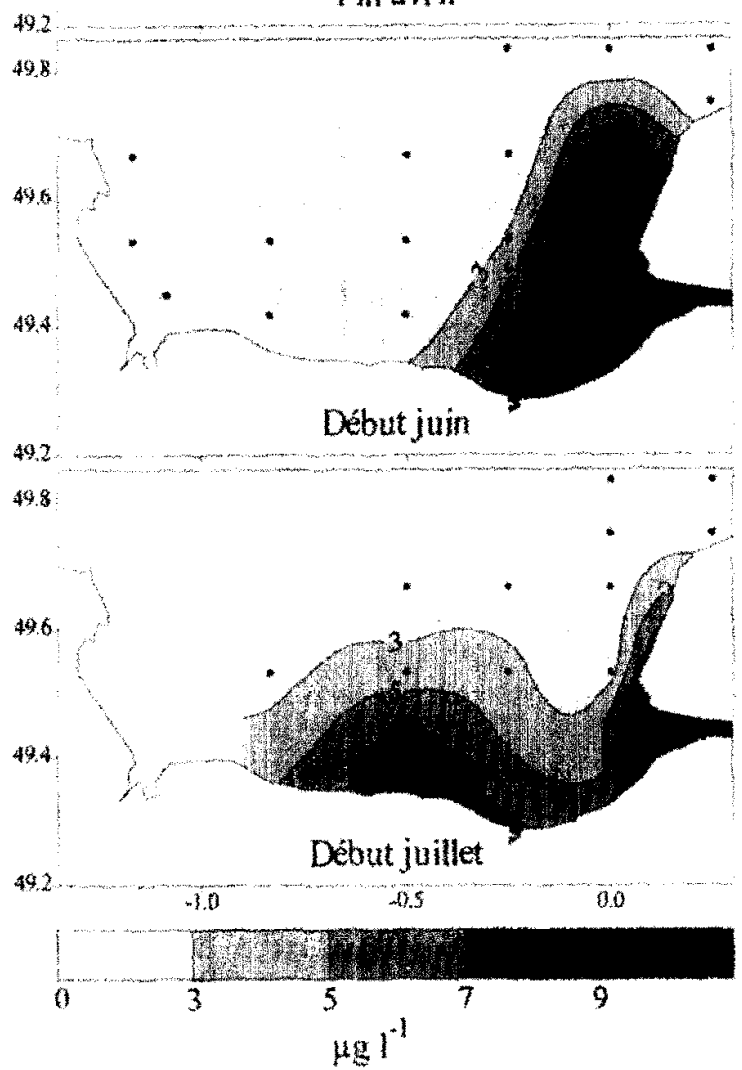

\section{P-orga sédimentaire}

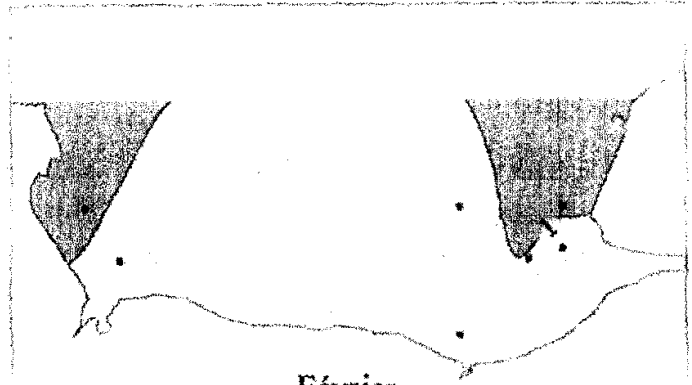

Février

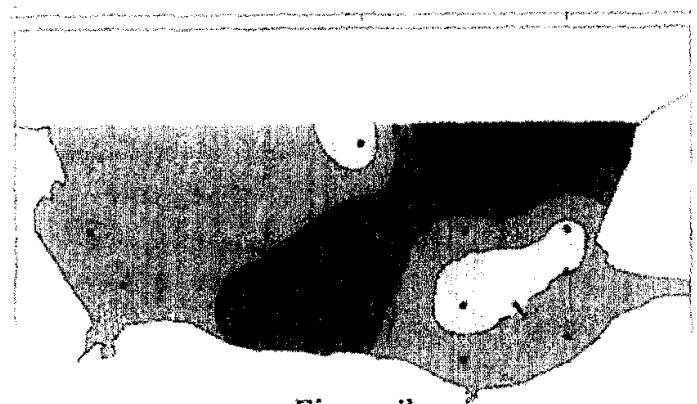

Fin avril

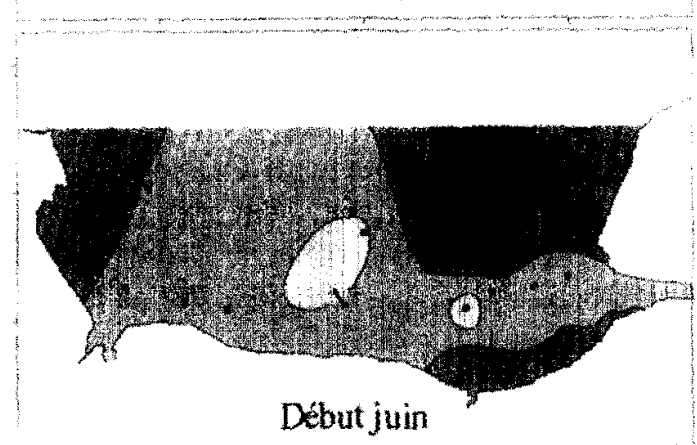

Début juin

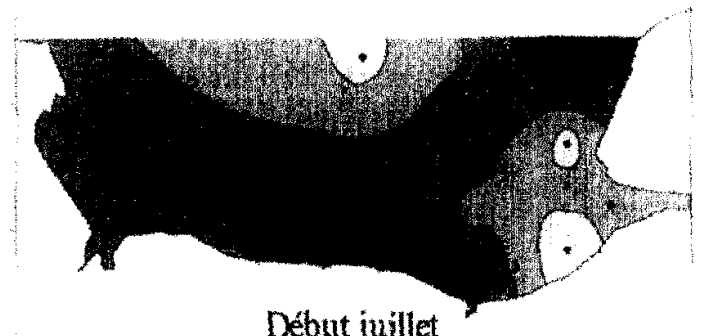

Début juillet

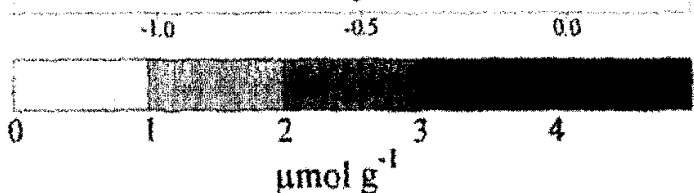

Figure 4. Distributions comparées de la chlorophylle a (surface, basse ner) et du phosphore organique sédimentaire de févirier à juillet 1992.

Figure 4. Comparative distribution panerns of chlorophyll a (surface, low tide) and of organic phosphons in sediment, from February to July 1992. 

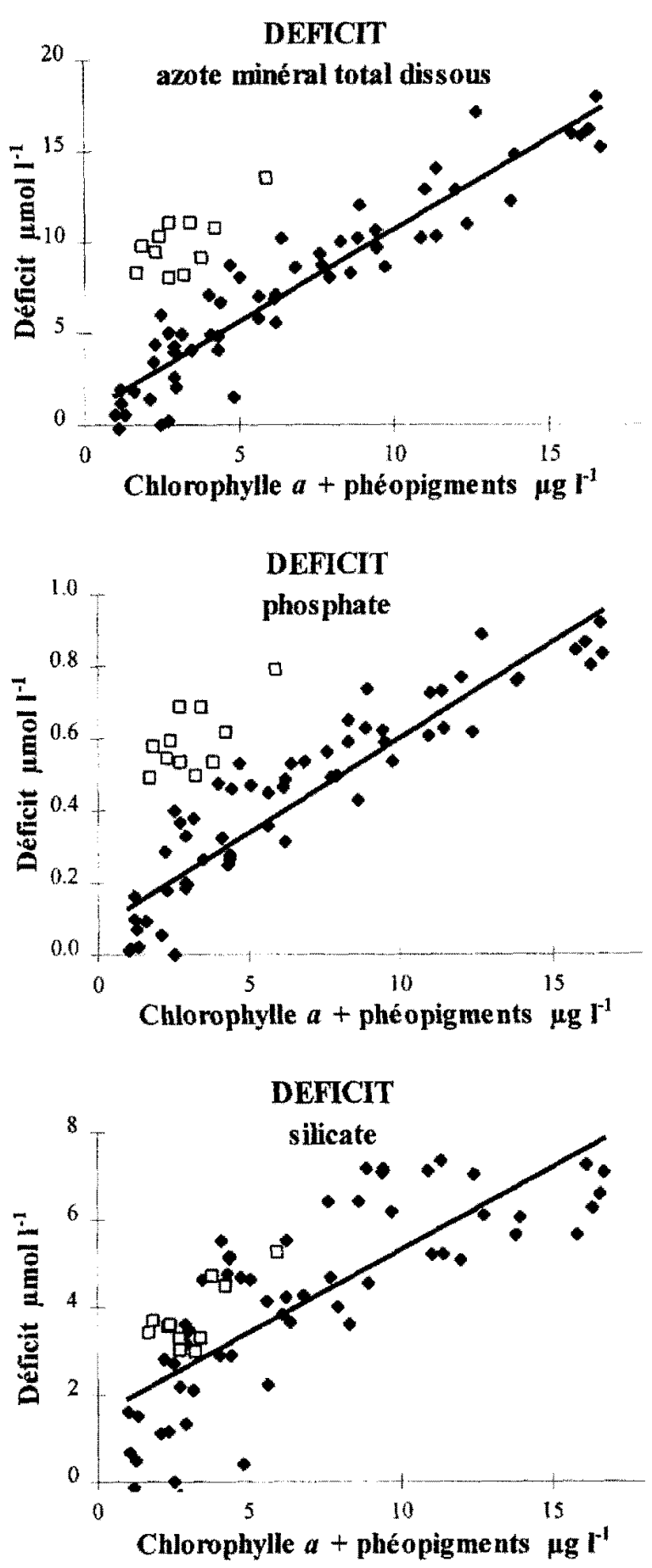

baie, pour des biomasses similaires. Il s'agit du nord de la baie, au-delà de la ligne Antifer-Barfleur $\left(49^{\circ} 40^{\prime}\right)$, où les pourcentages de phéopigments $(20-50 \%)$ sont, en outre, sensiblement plus élevés qu'aux autres stations marines $(<20 \%)$. Cette situation résulte très vraisemblablement de la poussée antérieure d'une population phytoplanctonique en voie de sédimentation. On remarquera qu'en silicate, le groupe de stations identifiées ne présente pas les fortes anomalies notées en azote et phosphore, mais seulement des valeurs plutôt élevées, en marge du nuage formé par les autres points. Le milieu se trouve en effet en conditions limitantes pour la poussée phytoplanctonique vis-à-vis du silicate $\left(<1 \mu \mathrm{mol} \mathrm{L}^{-1}\right)$. De ce fait, la consommation de silicate (et donc le déficit) n'a pu se poursuivre, contrairement à celles d'azote et de phosphore.

Après exclusion du groupe des stations marines identifiées ci-dessus, les corrélations linéaires entre les déficits de nutriments ( $\Delta \mathrm{N}, \Delta \mathrm{P}$ et $\Delta \mathrm{Si}$ ) et le marqueur de biomasse $\left(\mathrm{C}_{\mathrm{a}}\right)$ ont été calculées:

$$
\begin{aligned}
& \Delta N=0,57+1,00 C_{a} ;\left(n=64 ; r^{2}=0,88\right) \\
& \Delta P=0,075+0,053 C_{a} ;\left(n=64 ; r^{2}=0,79\right) \\
& \Delta S i=1,5+0,38 C_{a} ;\left(n=61 ; r^{2}=0,61\right)
\end{aligned}
$$

Cependant la relation entre les déficits de silicate et la biomasse pigmentaire n'apparaît pas linéaire sur l'ensemble du domaine d'étude; pour les fortes valeurs de pigments, les déficits de silicate plafonnent car les concentrations résiduelles de silicate sont considérées comme limitantes pour les diatomées, étant inférieures à $1 \mu \mathrm{mol} \mathrm{L}^{-1}$, moyenne des constantes de demi-saturation $\left(\mathrm{K}_{b}\right)$ présentées par Peeters et Peperzak [29]. En condition non limitante $\left(\mathrm{Si}>1 \mu \mathrm{mol} \mathrm{L}{ }^{-1}\right)$, la relation devient :

$\Delta \mathrm{Si}=0,87+0,50 \mathrm{C}_{\mathrm{a}} ;\left(\mathrm{n}=31 ; \mathrm{r}^{2}=0,50\right)$

Le rapport $\Delta \mathrm{N} / \mathrm{C}_{\mathrm{a}}$ tend vers $1,0 \mu \mathrm{mol} \mu \mathrm{g}^{-1}$ lorsque la biomasse phytoplanctonique augmente; ce rapport est conforme à la majorité des valeurs issues de la littérature [19]. Le rapport $\Delta \mathrm{P} / \mathrm{C}_{\mathrm{a}}$ tend vers $0,05 \mu \mathrm{mol} \mu \mathrm{g}^{-1}$. La valeur de $\Delta S \mathrm{Si} / \mathrm{C}_{\mathrm{a}}$, proche de $0,5 \mu \mathrm{mol} \mu_{\mathrm{g}}{ }^{-1}$, correspond aux valeurs usuelles [19]. Pour les concentrations en silicate inférieures à $1 \mu \mathrm{mol} \mathrm{L}^{-1}$, la pente de la relation $\Delta \mathrm{Si}$ $\mathrm{C}_{\mathrm{a}}$ s'abaisse à 0,2 ce qui traduit, compte tenu de la forte

Figure 5. Relations entre les déficits de nutriment et la biomasse phytoplanctonique (représentée par la somme chlorophylle a + phéopigments) pour la campagne de fin avril 1992. $\square$ : stations au nord de la baie (latitude $>49^{\circ} 40^{\prime}$ ). $\bullet$ autres stations, correspondant aux droites de régression représentées.

Figure 5. Relationships between nutrient uptake and phytoplanktonic biomass (as the sum of chlorophyll $a$ plus phaeopigments) at the end of April 1992. $\square$ : stations located in the north of the bay (latitude $>49^{\circ} 40^{\prime}$ ). 

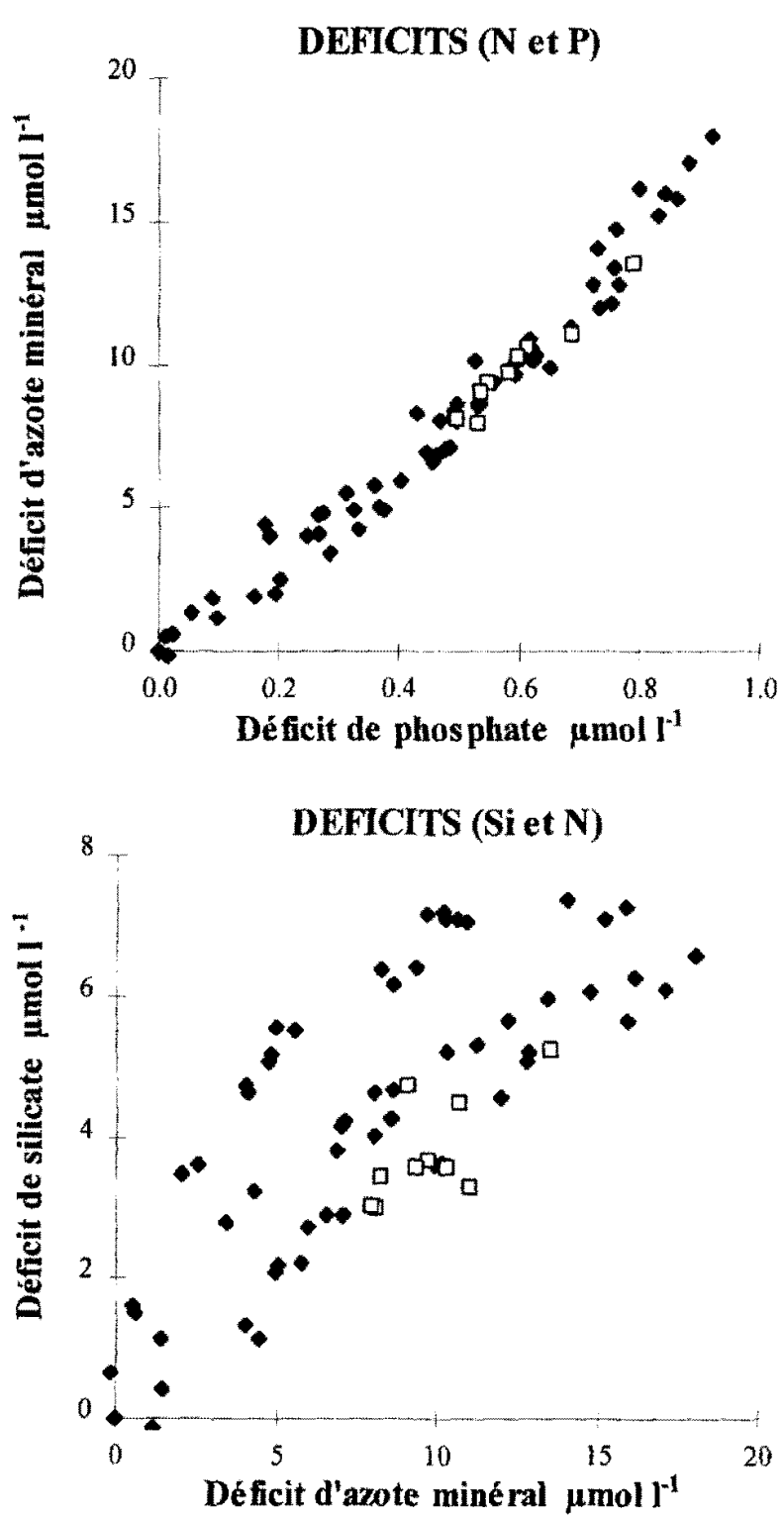

Figure 6. Relations entre les déficits d'azote minéral dissous, de phosphate et de silicate, pour la campagne de fin avril 1992. $\square$ : stations au nord de la baie (latitude $>49^{\circ} 40^{\prime}$ ). $\bullet$ autres stations.

Figure 6. Relationship between uptakes of nitrate, phosphate and silicate at the end of April 1992. $\square$ : stations located in the north of the bay (latitude $>49^{\circ} 40^{\prime}$ ). $\$$ : other stations.

domination des diatomées [36] une adaptation de ces cellules aux conditions limitantes en silicate [18].

Des tests ont montré que les pentes des relations ci-dessus, c'est-à-dire les rapports déficit en nutriments/biomasse pigmentaire, sont peu sensibles au choix des eaux de référence.

\subsubsection{Relations entre les déficits de nutriments}

La relation entre les deficits en azote minéral et les déficits en phosphate au cours de la poussée phytoplanctonique printanière d'avril 1992 est présentée sur la figure 6. La corrélation entre ces deux déficits $(\Delta N=$ $\left.-0.19+17,54 \Delta \mathrm{P} ; n=64 ; r^{2}=0,94\right)$ fournit une valeur moyenne du rapport N/P égale à 17,5, assez voisine de la valeur classique de 16 [31].

L'examen de la relation entre les déficits en silicate et en azote minéral permet d'individualiser un groupe de points correspondant à quatre stations, qui présentent pour des déficits en azote donnés, de plus forts déficits en silicate (figure 6). En excluant ces stations au comportement particulier, la corrélation entre les déficits de silicate et d'azote $\left(\Delta \mathrm{Si}=0,29+0,40 \Delta \mathrm{N} ; \mathrm{n}=44 ; \mathrm{r}^{2}=0,86\right)$ fournit une valeur moyenne du rapport atomique $\mathrm{Si} / \mathrm{N}$ égale à 0.4 . Morin [26] a montré les larges variations du rapport d'assimilation $\mathrm{Si} / \mathrm{N}$ selon les systèmes : 4 en Antarctique [20], I en ouest et sud-Bretagne [26], 0,5-1 dans le golfe de Gascogne [21], 0,3 à 0,5 dans l'upwelling marocain [35]. En zone tempérée, les valeurs semblent limitées à une gamme de 0,3 à 1. En comparaison, pour la composition de trois espèces de diatomées en conditions non limitantes, Harrison et al. [18] trouvent des rapports $\mathrm{Si} / \mathrm{N}$ de 0,43 à 0.55 . Les rapports d'assimilation donnés par Billen [11] en mer du Nord sont de 0,38-0,40. Quant aux quatre stations où le rapport des déficits $\triangle \mathrm{Si} / \mathrm{DN}$ est plus élevé, elles sont situces dans le panache de dilution des eaux douces, où la salinité s'élève de 33 à 34 et où les turbidités deviennent inférieures à $1 \mathrm{NTU}$ (environ $2 \mathrm{mg} \mathrm{L}^{-1}$ de matières en suspension). Le long de ce gradient de dilution les teneurs en chlorophylle $a$ s'élèvent de 2 à $10 \mu \mathrm{gL}^{-1}$, signe d'une forte production primaire favorisée par la plus faible turbidité et par un temps de séjour accru des cellules dans ces masses d'eaux. Parallèlement, les teneurs en silicate baissent le long de ce gradient, de $6 \mu \mathrm{mol} \mathrm{L} \mathrm{L}^{-1}$ à $0,6 \mu \mathrm{mol} \mathrm{L} \mathrm{L}^{-1}$, devenant limitantes contrairement à celles d'azote $\left(19 \mu \mathrm{mol} \mathrm{L}^{-1}\right)$ et de phosphate $\left(1 \mu \mathrm{mol} \mathrm{L} \mathrm{L}^{-1}\right)$. Le rapport entre les déficits de silicate et les déficits d'azote minéral présente, dans cette zone, des valeurs élevées de 1,7 à 0,65 . Tout se passe comme s'il y avait dans ces eaux un pompage très actif du silicate par les diatomées en phase de croissance.

\section{SYNTHESE ET CONCLUSION}

Bien que limitée dans le temps, cette étude permet de constater qu'en première approximation les flux de nutri- 
ments de la Seine sont peu affectés par la traversée de l'estuaire, quelle que soit la saison: les apports fluviatiles se répercutent intégralement sur la baie, après un temps de séjour moyen dans l'estuaire n'excédant la dizaine de jours que lors des étiages sévères. Le calcul des flux effectué en Seine à Caudebec permet d'estimer valablement les apports à la baie.

La Seine a des concentrations en azote total élevées ef relativement stables au cours de l'annee (environ $600 \mu \mathrm{mol} \mathrm{L}^{-1}$ en moyenne), dont près de $90 \%$ sous forme minérale dissoute, immédiatement assimilable par le phytoplancton. La Seine est aussi particulièrement riche en phosphate dissous qui, issu majoritairement de sources ponctuelles sur le bassin versant, présente de fortes variations $\left(10-50 \mu\right.$ mol $\left.\mathrm{L}^{-1}\right)$ inverses de celles du débit. Le phosphore particulaire, dont les différentes formes ont été antérieurement déterminées [1]. constitue globalement $28 \%$ du phosphore total : seule la moitié de cet apport est considérée comme biodisponible à cout terme. Le silicate quasiment épuisé en été du fait de la consommation phytoplanctonique fluviale, peut atteindre $200 \mu \mathrm{mol} \mathrm{L}^{-1}$ lors des crues hivernales. Durant la période d'étude (1992-1994), le calcul des flux annuels révèle, selon que lannée est sèche ou humide, des apports très différents en azote minéral dissous (de 80000 à $130000 \mathrm{t} \mathrm{a}^{\prime \prime}$ ) et en silicium dissous (de 20000 a $77000 \mathrm{t} \mathrm{a}^{-1}$ ), alors que les flux de phosphore dissous restent relativement stables $\left(6400\right.$ à $\left.8400 \mathrm{t} \mathrm{a}^{-1}\right)$.

Lienrichissement de la baie en nutriments dissous suit le gradient des isohalines, orientés du sud-est vers le nordouest, montrant ainsi l'influence continentale tout au long de l'année.

Dans le sédiment, le phosphate lié au fer et à l'aluminium et le phosphate échangeable montrent l'influence des particules fines d'origine estuarienne dont la dérive vers le nord crée un gradient décroissant est-ouest bien marqué et stable durant l'année. Les phosphates de calcium on une répartition plus uniforme du fait d'une proportion significative de sédiments marins calcaires dans la baie.
Le phosphore organique des sédiments superficiels, peu marqué par les apports estuariens, est nettement influencé par la production primaire, mais il ne s'accumule pas durablement, signe d'une régénération rapide avec retour au milieu pélagique. Si l'on retient que seules les formes organique et échangeable du phosphore sont potentiellement biodisponibles, le matériel particulaire issu de lestuaire ne paraît pas enrichir notablement les sédiments de la baie.

Considérant le développement printanier du phytoplancton, on note qu'il débute au large, puis s'établit au centre de la baie, en limite du panache de la Seine, où les temps de résidence sont suffisants et les eaux moins turbides. En été la zone de forte concentration en chlorophylle $a$ (> $20 \mu \mathrm{g} \mathrm{\textrm {L } ^ { - 1 }}$ ) se déplace vers les côtes sud-est de la baie en tormant un gradient décroissant côte-large. Des valeurs supérieures à $40 \mu \mathrm{g} \mathrm{L}^{-1}$ se rencontrent chaque année, signe d'un milieu cutrophe.

L'étude des déficits en nutriments en période de poussée planctonique a permis d'établir des rapports entre la consommation de nutriments et la biomasse pigmentaire (chlorophylle $a+$ phéopigments) qui sont de $1 \mu \mathrm{mol} \mu \mathrm{g}^{-1}$ pour l'azote, de $0,05 \mu \mathrm{mol} \mu \mathrm{g}$ pour le phosphore, et de $0.5 \mu \mathrm{mol} \mu \mathrm{g}^{-1}$ pour le silicate. Les rapports atomiques de consommation des eléments nutritifs sont de 17.5 pour $\mathrm{N} / \mathrm{P}$ et de 0,4 pour $\mathrm{Si} / \mathrm{N}$; ils sont peu différents des valeurs moyennes issues de la littérature.

\section{Remerciements}

Cette étude a été financée par le Programme National d'Océanographie Côtière (thème « eutrophisation »). Les auteurs remercient Alain Ménesguen pour son appui dans l'organisation des campagnes à la mer, ainsi que Annie Chapelle et l'équipage du N.O. Thalia pour leur aide lors de ces campagnes, el André Ficht pour la fourniture, sur support informatique, des données de la cellule antipollution de la Seine.

\section{RÉFÉRENCES}

[1] Aminot A., Guillaud J.F., Andrieux F., Spéciation du phosphore et apports en baie de Seine orientale, Oceanol. Acta 16 (5-6) (1993) 617-623.
12| Aminot A.. Guillaud J.F., Kérouel R. La baie de Seine: hydrologie, nutriments et chlorophylle (1978-1994), Repères Océan 14 (1997) 148 p. 
[3] Aminot A., Kérouel R., Matvais J.L., Les éléments nutritifs en baie de Seine, la baie de Seine, Ifremer, actes de colloques, 4 (1986) $289-295$

[4] Andrieux-Loyer F., Les formes de phosphore particulaire et sédimentaire en enviromnement côtier, méthodes d'analyse, biodisponibilité, échange, thèse univ. Bretagne occidentale (1997) $310 \mathrm{p}$.

15] Armstrong F.A.J., Butler E.I., Boalch G.T., Hydrographic and nutrient chemistry surveys in the western English Channel during 1965 and 1966, J. mar. biol. Ass. U.K. 54 (1974) 895 914.

[6] Avoine J., Lestuaire de la Seine: sédiments et dynamique sédimentaire, thèse univ. Caen (1981) $236 \mathrm{p}$.

17] Avoine J., Evaluation des apponts fluviatiles dans l'estuaire de la Seine, la baie de Seine, Ifremer, actes de colloques, 4 (1986) $117-124$.

[8] Avoine J., Dubrulle L., Larsonneur C., La dynamique sédimentaire dans les estuaires de la baie de Seine-Conséquences sur l'environnement, la baie de Seine, lfremer, actes de colloques, 4 (1986) 183-192

[9] Avoine J., Boust D., Guillaud J.-F., Flux et comportement des contaminants dissous et particulaires dans l'estuaire de la Seine, rapp. P.-v. Réun. Cons. int. Explor. Mer 186 (1986) $392-411$.

[10] Belin C., Berthomé J.-P., Lassus P., Dinolagellés toxiques et phénomènes d'eaux colorées sur les côtes françaises: évolution et tendances entre 1975 et 1988, Hydroecol. Appl $1-2$ (1989) $3-17$

[11] Billen $G$, Coastal eutrophication in a global perspective, in : Symoens et al. (éd.), Biological indicators of global change Symposium (Bruxelles, 7-9 mai 1992). Royal Academy of Overseas Science, Bruxelles, 1993, pp. 17-34.

[12] Boust D., Les métaux-traces dans l'estuaire de la Seine et ses abords, thèse univ. Caen (1981) $230 \mathrm{p}$.

[13] Cellule antipollution de la Seine. Suivi de la qualité des eaux de la Seine à l'aval de Poses, bilan annuel. Ministère de l'environnement, service maritime de la Seine Maritime ( $3^{\circ}$ section), documents non paginés (1977-1994).

[14] Folkard A.R.. Jones P.G.W., Hydrographic observation in the southern North Sea during 1961 and 1962, with particular reference to the distribution of nutrients salts, Fish. Res. techn. Report 48, MAFF-DFR (1978) 44 p.

[15] Goujon R., Dupont J.P.. Meyer R., L'estuaire de la Seine. Compte rendu du colloque national : estuaires et deltas : des milieux menacés?. Bègles, 25 juin 1992, 1-8.

[16] Guilhaud J.F, Les flux de sels nurritifs dans restuaire de la Seine (France) ; rôle et importance du bouchon vaseux au cours du mélange estuarien, Can. J. Fish. Aquat. Sci. 40 (suppl. 1) (1983) 180-187.

[17] Guillaud J.F., Menesguen A.. Modélisation sur vingt ans (1976-1995) de la production phytoplanctonique en bate de Seine, Oceanol. Acta 21 (6) (1998)

[18] Harrison P.J., Conway H.L., Holmes R.W., Davies C.O., Marine diatoms grown in chemostats under silicate or ammo- nium limitation. 3, Cellular chemical composition and morphology of Chaetoceros debilis, Skeletonema costatum and Thalassiosira gravida, Mar. Biol 43 (1) (1977) 19-31.

[19] Jorgensen S.E., Handbook of environmental data and ecological parameters, Intemational Society for Ecological Modelling-[ISEM](ed.), Vaerlose (DK) (1979) $1162 \mathrm{p}$.

[20] Le Corre P., Minas H.J, Distribution et évolution des éléments nutritifs dans le secteur indien de l'Océan antarctique en fin de période estivale, Oceanol. Acta 6 (1983) 365-381.

[21] Le Corre $P$., Tréguer $P_{*}$, Contribution à l'étude de la matière organique dissoute et des sels nutritifs dans l'eau de mer. Caractéristiques chimiques du golfe de Gascogne et des upwellings cotiers de l'Afrique do nord-ouest. thèse univ. Bretagne occidentale (1976) $490 \mathrm{p}$.

[22] L'Helguen $S_{\text {. }}$ Absorption et régénération de l'azote dans les ecosystèmes pélagiques du plateau continental de la Manche occidentale, relation avee le régime de mélange vertical des masses deaux : cas du front thermique d'Ouessant, thèse univ. Bretagne occidentale (1991) $212 \mathrm{p}$.

[23] Loder T.C. Reichard R.P., The dynamic of conservative mixing in estuaries, Estuaries 4 (1) (1981) 64-69.

[24] Lorenzen C.J., Détermination of chlorophyll and phaeopigments: spectrophotometric equations, Limnol. Oceanogr. 12 (1967) $343-346$

[25] Menesguen A., Guillaud J.-F., Aminot A., Hoch T. Modelling the eutrophisation process in a river plume: the Seine case study (France), Ophelia 42 (1995) 205-225.

[26] Morin P. Évolution des eléments nutritifs dans les systèmes frontaux de l'Iroise: assimilation et réténération; relation avec les structures hydrologiques ef les cycles de développement du phytoplancton. thèse univ. Bretagne occidentale (1984) $220 \mathrm{p}$.

[27) Morin P., Le Corre P.. Le Fèvre J., Assimilation and regeneration of nutrients off the western coast of Brittany, J. mar, biol. Ass. U.K. 65 (1985) 677-695.

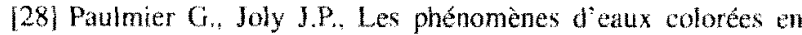
baie de Seine, Ifremer, actes de colloques, 4 (1986) $477-484$.

[29] Peeters J.C.H., Peperzak L., Nutrient limitation in the North Sea: a bioassay approach, Neth, J. Sea Res. 26 (1) (1990) 61 73 ,

[30] Psenner R., Boström B., Dinka M., Petterson K., Pucsko R., Sager M., Fractionation of phosphorus in suspended matter and sediment, Arch. Hydrobiol. Beih. Ergeb. Limnol. 30 (1988) 98-103.

[31) Redfield A.C., Ketchum B.H., Richards F.A., The influence of organisms on the composition of sea water, in: The Sea, V.2, Hill M.N., Interscience (1963) 26-77.

[32] RNO Surveillance du milieu marin. Travaux du RNO, édition 1992-1993 (1993) $32 \mathrm{p}$.

[33] Tappin A.D., Studies of trace metals in shelf waters of the British Isles, thèse univ. Southampton (1988) $279 \mathrm{p}$.

[34] Tréguer P. Le Corre P.. Manuel d'analyse des sels nutritif, dans l'eau de mer, univ. Bretagne occidentale (1975) $110 \mathrm{p}$. 
NUTRIMENTS ET CHLOROPHYLLE EN BAIE DE SEINE

135) Tréguer P., Le Corre P. The ratio of nitrate, phosphate and silicate during uptake and regeneration phases of the Moroccan upwelling regime, Deep-Sea Res. 26 (1979) 163-184.

[36] Videau C., Ryckaen M. L'Helguen S., Phytoplancton en baje de Seine (France), Influence du panache de la Seine sur la production primaire, Oceanol. Acta 21 (6) (1998) 907-921.
[37] Walling D.E., Webb W., Estimating the discharge of contaminants to coastal waters by rivers: some cautionary comments, Mar. Poll. Bull. 16 (12) (1985) 448-492.

[38] Williams J.D.H., Jaquet J.M., Thomas R.L., Forms of phosphorus in the surficial sediments of Lake Erie, J. Fish. Res. Bd. Can. 33 (1976) $413-429$. 